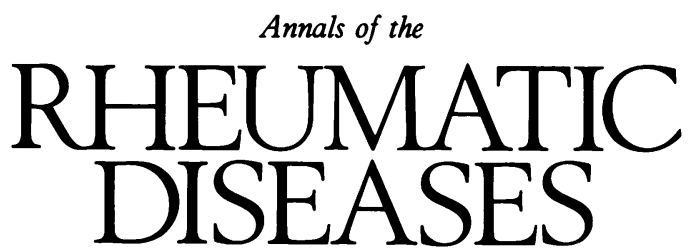

Leader

\title{
Coping with rheumatoid arthritis
}

Some patients with severe rheumatoid arthritis (RA) as measured radiographically or by other clinical assessments appear not to be very disabled, whereas others with seemingly mild disease appear to be severely debilitated. This phenomenon has been apparent to many rheumatologists and similar findings have been reported in other diseases. The poor correlation between measures of disease state and disability underlines the problem of understanding the impact of RA on the individual patient. ${ }^{1}$ With a few exceptions ${ }^{2}$ most studies of the natural history of RA have focused on disease and biological factors, and have ignored psychological factors which may act to modify the extent of the symptoms reported, the course of the illness, and the patient's feelings of wellbeing.

One of the potentially useful concepts to bridge the gulf of our understanding between disease and disability and psychological wellbeing is coping. A typical definition of coping is 'the cognitive, behavioural and emotional efforts individuals exert to manage specific external and/or internal demands'. ${ }^{3}$ Coping responses are considered to result from the patient's assessment of the stresses that they

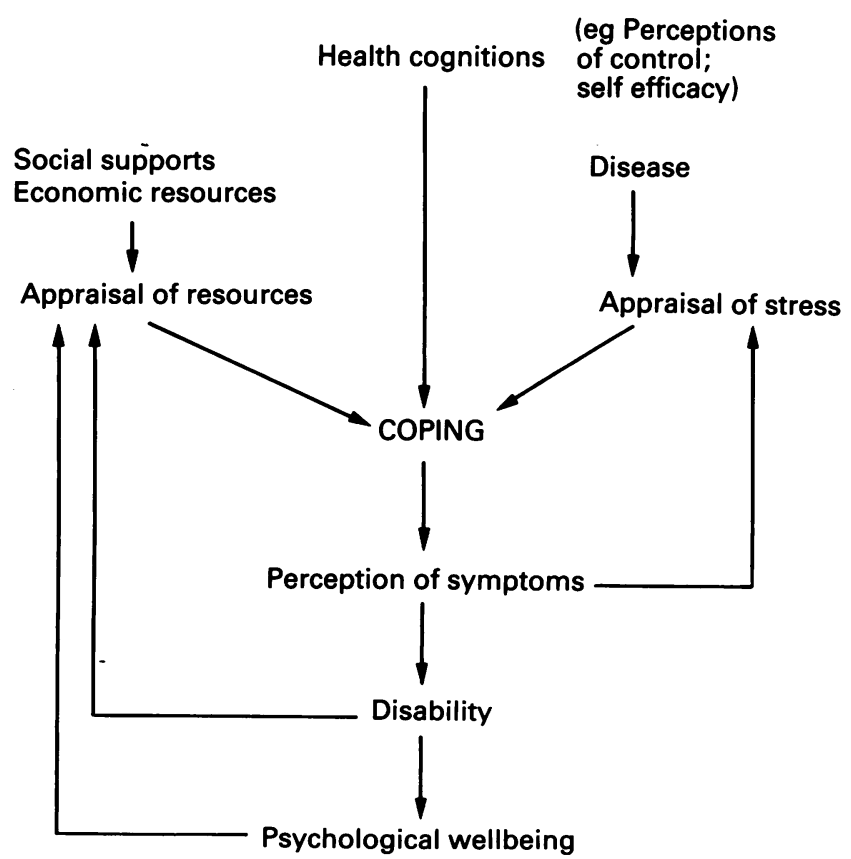

Schematic diagram of the influences on, and of coping strategies in, chronic illness. face and the resources available. The stress may threaten the patient physically or psychologically, as in the case of a threat to their self esteem. By examining the particular strategies or combination of strategies that patients use it becomes possible to determine which lead to a favourable outcome. This in turn lets us know which coping strategies to foster in patients.

The figure shows how coping may act as an intervening factor between disease and its effects and the possible influences on and of coping. In this model the effects of the disease are mediated by different patterns of coping which result in different symptoms and are manifested in different levels of disability, which together establish different levels of psychological outcome.

Once patients are diagnosed as having RA they face a range of stresses, including adjusting to medical treatment and uncertainty about the course of the disease. The symptoms of pain and stiffness may well have been present before diagnosis but in the long term these may increase and lead to restricted movement and disfigurement. This in turn may limit the ability to fulfil work and social roles and therefore have an effect on other family members. The patient uses coping strategies to minimise the impact of the disease. In many instances they may have to choose between conflicting demands. For example, an attempt to deal adequately with a work role may require a strategy to ignore or play down pain. The coping strategies they apply may involve actions or particular ways of thinking or feeling, or both. Coping takes many forms and some common examples are given in the table.

Coping strategies may be directed towards reducing the impact of pain and stiffness, the extent of disability, and in some instances towards making the subject feel better. Psychological wellbeing is an important domain as patients with RA, or for that matter other chronic illnesses, have an increased risk of psychological difficulties and lowered self esteem. Studies have shown that with RA the

Examples of coping strategies

Information seeking

Reorganise routines

Denial

Wish fulfilmen

Distraction

Seek social support

Express emotions

Seek spiritual support

Reinterpretation

Selective comparison 
combination of pain, stiffness, disability, and social restrictions results in significantly increased levels of depressed mood. ${ }^{4-6}$ Although disability has been found to be the most important variable in determining psychological adaptation in RA, a number of other factors such as social contacts and economic resources appear to be important. ${ }^{4}$ These findings imply that for most subjects the stresses of RA are wide ranging and have an effect on their psychological wellbeing.

Rheumatoid arthritis often takes some time to develop and subjects have the opportunity to adjust their behaviour and coping strategies. Time has two conflicting effects. On one hand, increasing time with the disease leads, in most patients, to higher levels of disability, which in turn lead to lower levels of psychological wellbeing. ${ }^{4}{ }^{8}$ On the other hand, time may also lead to a higher level of psychological adaptation as patients learn to adjust to the disease and perhaps alter their expectations. In a study of 158 adult patients with RA the effects of time were examined independently of the level of disability. ${ }^{4}$ When the level of disability was controlled for statistically, time since diagnosis was associated with improved psychological wellbeing. Specifically, those patients with RA who had the disease the longest were better adjusted. These findings suggest that when the influence of disability is 'removed', it exposes the psychological work that may be performed over time.

Social comparison is one of many forms of coping that may have particular importance in understanding the adaptation that some patients make to seemingly severe disease and disability. It occurs particularly when the means to change the situation are not available. ${ }^{9}$ By this process some patients with more advanced disease may compare themselves with others in a worse state and, in this manner, preserve their psychological wellbeing. Studies of RA have shown that patients do predominantly tend to make comparisons with those in a worse state. ${ }^{1011}$ This coping strategy, known as downward comparisons, was associated with better psychological wellbeing. Severe disease and worsening disease were particularly associated with comparisons with patients in a worse state. ${ }^{11}$

Several studies ${ }^{12-14}$ have been conducted which have adapted the Ways of Coping Questionnaire, a widely used questionnaire designed to assess responses to stress, for use with patients with RA. ${ }^{15}$ They have all suggested that coping strategies such as wishful thinking, a form of fantasising about the illness, lead to poorer psychological wellbeing. In contrast, cognitive restructuring, which involves attempting to alter the patient's views or thoughts about the disease to be more positive, leads to improved outcomes with regard to psychological wellbeing. It is important that these studies have used different patient groups and have been conducted at different times since the diagnosis of the disease and yet all tend to support the improved outcome with cognitive restructuring. Two studies also found that seeking information about the disease was also associated with improved outcome. ${ }^{12} 14$

Our own research has focused on patients with established disease and has adopted a methodology which enables the whole range of possible coping strategies and the extent to which they are used to be examined. ${ }^{16}$ Thus, for example, a patient who often uses distraction and spiritual relief as coping strategies should be distinguished from a patient who uses distraction but strongly rejects any spiritual coping strategies. A large sample of patients attending an outpatient rheumatology clinic were assessed on a 36 item questionnaire about coping with RA. Four distinct groups who attempted to cope with their disease in different ways were identified. One of these consisted of patients who attempted to make little accommodation to their illness. They were characterised by being open in the manner in which they attempted to deal with the stresses of arthritis. They did not use denial, wish fulfilment, distraction, prayer, or religion. They did confront their disease, refused to reorganise their routines, engaged in physical activity, and expressed their feelings. Although the four groups were almost indistinguishable on demographic, clinical, and laboratory measures of arthritis, the group using active coping strategies was found to report lower levels of pain and stiffness in addition to disability. They also had significantly higher levels of psychological wellbeing. These findings suggest that there are different groups of patients who have similar levels of disease but who cope with their illness in different ways, and that these different strategies of coping have an effect on the reporting of symptoms, disability, and psychological wellbeing.

The research on coping with RA has led to the development of interventions to improve outcome. Several early intervention studies have been built on providing patients with information about arthritis to improve their knowledge of the disease. ${ }^{17}$ These were based on the premise that information leads to knowledge, which in turn leads to behavioural changes and thus to improved outcome. These educational interventions have since been broadened to consider physical and psychosocial outcomes and have included an examination of psychological variables such as coping. ${ }^{18}$ This work is still in its infancy and much research remains to be done in this area to examine whether coping strategies are easy or difficult to change and which coping strategies are most appropriate to encourage. It is also important to devise methods for the assessment of coping to incorporate these into clinical practice and to identify which patients would best benefit from intervention.

Academic Department of Psychiatry,

STANTON NEWMAN

Wolfson Building

Riding House Street,

London W1N $8 A A$

United Kingdom

1 Bijlsma J, Huiskes C, Kraaimaat F W, Vanderveen M J, Huber-Bruning O. Relation between patients' own health assessment and clinical and laboratory findings in rheumatoid arthritis. 7 Rheumatol 1991; 18: 650-3.

2 Lorish C D, Abraham N, Austin J, Bradley L A, Alarcon G S. Disease and psychosocial factors related to physical functioning in rheumatoid psychosocial factors related to phys

3 Lazarus R S, Folkman S. Stress, appraisal and coping. New York: Springer, 1984

4 Newman S, Fitzpatrick R, Lamb R, Shipley M. The origins of depressed mood in rheumatoid arthritis. F Rheumatol 1989; 16: 740-4

5 Anderson K O, Bradley L A, Young L D, McDaniel L K. Rheumatoid arthritis: review of psychological factors related to etiology, effects and treatment. Psych Bull 1985; 98: 358-87.

6 Zaphiropoulos G, Burry H C. Depression in rheumatoid disease. Ann Rheum Dis 1974; 33: 132-5.

7 Fitzpatrick R, Newman S, Lamb R, Shipley M. Social relationships and psychological well being in rheumatoid arthritis. Soc Sci Med 1988; 27: 399-403.

8 Revenson T A, Felton B J. Disability and coping as predictors of psychological adjustment to rheumatoid arthritis. $\mathcal{F}$ Consult Clin Psychol psychological ad $1989 ;$ 57: $344-8$.

9 Wills T A. Downward comparison as a coping mechanism. In: Snyder C R, Ford C E, eds. Coping with negative life events. New York: Plenum Press, 1987.

10 Affleck G, Tennan H, Pfeiffer C, Fifield J. Social comparison in rheumatoid arthritis: accuracy and adaptational significance. F Soc Clin Psychol 1988; 6: 219-34.

11 DeVellis R, Holt K, Renner B, et al. The relationship of social comparison to rheumatoid arthritis symptoms and affect. Basic Appl Soc Psychol 1990; 11: $1-18$.

12 Felton B J, Revenson T A, Hinrichsen G A. Stress and coping in the explanation of psychological adjustment among chronically ill adults. Soc Sci Med 1984; 18: 889-98.

13 Parker J, McRae C, Smarr K, et al. Coping strategies in rheumatoid arthritis. F Rheumatol 1988; 15: 1376-83.

14 Manne S, Zautra A J. Spouse criticism and support: their association with coping and psychological adjustment among women with rheumatoid arthritis. F Pers Soc Psychol 1989; 56: 608-17.

15 Folkman S, Lazarus R S. An analysis of coping in a middle aged community sample. भ Health Soc Behav 1980; 21: 219-39.

6 Newman S, Fitzpatrick R, Lamb R, Shipley M. Patterns of coping in rheumatoid arthritis. Psychology and Health 1990; 4: 187-200.

17 Lorig K, Konkol L, Gonzalez V. Arthritis patient education: a review of the literature. Patient Education and Counselling 1987; 10: 207-52.

18 Daltroy $L$, Liang M. Advances in patient education in rheumatic disease. Ann Rheum Dis 1991; 50: 415-7. 